\title{
A study on major design elements of tracking-type floating photovoltaic systems
}

\author{
Young-Kwan Choi ${ }^{a^{*}}$, Nam-Hyung Lee ${ }^{\mathrm{a}}$, An-Kyu Lee ${ }^{\mathrm{a}}$, Kern-Joong Kim ${ }^{\mathrm{b}}$ \\ ${ }^{a}$ K-Water, 462-1 Jeonmin-Dong Yuseong-Gu Daejeon, 305-730, Republic of Korea \\ ${ }^{b}$ Chungnam National Univ., Gung-dong Yuseong-gu Daejeon, 305-764, Republic of Korea
}

\begin{abstract}
A floating PV system results from the combination of photovoltaic power plant technology and floating technology. K-Water has installed a $100 \mathrm{~kW}$ floating PV system on the water surface on Hapcheon dam reservoir in October 2011 and has been operating it since then. After successfully installing the $100 \mathrm{~kW}$ floating PV system, K-Water additionally installed a $500 \mathrm{~kW}$ floating PV system on another location nearby in July 2012. The electricity generated by the two floating PV systems installed in Hapcheon dam reservoir is creating profits by being sold to the national power grid. In this article, taking a step further from such fixed-type floating PV, the basic concept of $100 \mathrm{~kW}$ tracking-type floating PV and the application plan for the tracking algorithm and the rotation mechanism of structure which is a major design element were explained. As the first case that can maximize the power generation efficiency of PV internationally, it is expected that this study will be utilized as a primary guide for future development of tracking type PV system.
\end{abstract}

Keywords:Photovoltaic system, floating PV system, tracking-type floating PV

\section{Introduction}

Recently, the photovoltaic market is expanding due to the introduction of RPS(Renewable Energy Portfolio Standard), and studies on alternatives to the land PV are actively being carried out due to lack of installation sites. Both internally and externally, there are not many cases where floating PV system is installed and operates on the surface of dams or reservoirs. The interest in floating PV is rapidly increasing as the floating PV REC weighted value, which used to apply 1.0 by 2012 , was newly enacted in 2013 as 1.5, the same level as BIPV(Building Integrated Photovoltaic System). This is because the amount of power generation is greater on water surface than ground surface so that an efficient energy production can be achieved. Also, recently the interest in tracking-type floating PV which can generate $20 \%$ improved amount than floating PV is also rising. This article intends to explain the basic concept of $100 \mathrm{~kW}$ tracking-type floating PV that K-Water intends to develop, and also the application plan for the tracking algorithm and the rotation mechanism of structure which is a major design element [1].

\section{Concept of Floating PV System}

\subsection{Floating PV system}

A developed floating PV system results from the combination of PV plant technology and floating technology. This fusion is a new concept for technology development. As a new generation technology, it can replace the previous PV plant that installed on top of existing woodland, farmland and buildings. The floating PV plant consists of a floating system, mooring system, PV system and underwater cables [2]-[4].

\footnotetext{
* Manuscript received May 14, 2013; revised July 24, 2013.

Corresponding author. Tel.: +82-42-870-7661; E-mail address: music@kwater.or.kr.
} 


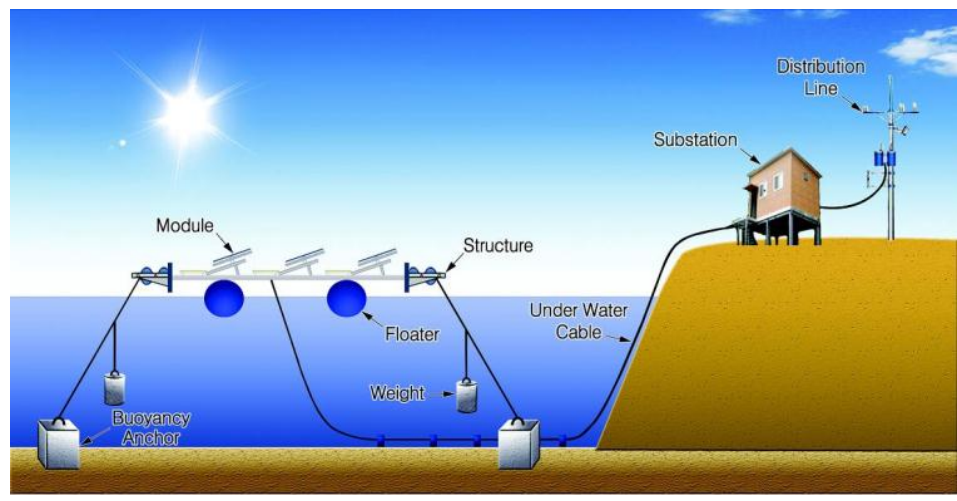

Fig. 1. Floating PV plants outline.

a) Floating system: a floating body (structure + floater) that allows the installation of the PV module.

b) Mooring system: can adjust to water level fluctuations while maintaining its position in a southward direction.

c) PV system: PV generation equipment, such as electrical junction boxes, that are installed on top of the floating system.

d) Underwater cable: transfers the generated power from land to the substation.

\subsection{Tracking-type floating $P V$ system}

PV is divided into fixed-type where the angle of PV module is fixed at a certain angle and trackingtype where the azimuth and altitude of the sun is tracked to receive the sunlight perpendicular to the module surface. The tracking-type PV is a high-efficiency generation system that produces a greater amount of electricity by adding real-time sun-tracking function to the PV module. In general, it is known that on the ground, the power generation of a dual-axis tracking-type is 30\% greater than a fixed-type.

Tracking-type PV system is designed to detect the existence of effective PV to track the location of the sun with solar sensor, and automatically track the sun with precision by program even when it is cloudy or rainy. For this, a separate mechanical device is used to rotate the PV module, but the number of unit $\mathrm{PV}$ modules that can be rotated at once is limited, and require a great deal of effort in maintenance due to frequent malfunctions, etc.

Due to such maintenance issues, there are controversies as to the economic value of tracking-type PV, but in the case of Korea which lacks renewable energy sources, a persistent study on tracking-type is necessary to utilize the limited resources to the maximum.

Tracking-type floating PV system combines "floating" PV system and "tracking-type" PV system. The combination of such two conditions should be considered for the influence on tracking system by the environmental condition of floating on water surface.

The tracking-type system used on the ground has limitations as the number of PV modules that can be operated through a single tracker is limited (below $3 \mathrm{~kW}$ ) and the utility is also limited due to frequent malfunctions. Due to such factors, the structure must be designed with cantilever at a single rotation point, and as the cantilever length is limited due to the influence of self-weight or external force, there is the weakness that the cost of the structure raises as the unit module capacity increases.

In floating PV, the external forces including self-weight of the structure is transferred to the water through the buoyancy in the gravitational direction, so the length of the structure that can resist the gravitational direction can be designed with relatively more freedom. Also, as it floats on the water, it can rotate with a small amount of energy even if the unit rotation capacity is large (over $20 \mathrm{~kW}$ ), the structure can be made simpler than ground tracking-type if tracking-type is applied to floating PV system, thus reducing malfunction risk and increasing efficiency. The features for each form were compared and shown in the following Table 1. 
Table 1. Characteristics of fixed-type and tracking-type floating PV

\begin{tabular}{|c|c|c|}
\hline Classification & Form & Characteristics \\
\hline $\begin{array}{l}\text { Fixed-Type } \\
\text { Floating PV }\end{array}$ & 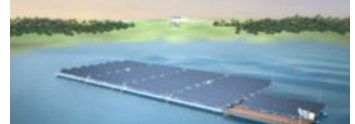 & $\begin{array}{l}\text {-Convenient structure design and construction } \\
\text {-Smaller generation amount than tracking-type } \\
\text {-Precise mooring construction for direction fixation }\end{array}$ \\
\hline $\begin{array}{l}\text { Tracking-Type } \\
\text { Floating PV }\end{array}$ & 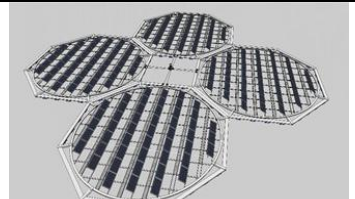 & $\begin{array}{l}\text {-Rotates structures considering the floating characteristics } \\
\text {-Unit rotation capacity can be greater } \\
\text {-Increased generation amount than fixed-type } \\
\text {-Relatively harder construction than fixed-type } \\
\text {-Horizontal rotation-type and dual-axis type with tilt-variable added }\end{array}$ \\
\hline
\end{tabular}

\section{Design of Tracking-Type Floating PV}

\subsection{Design concept of tracking-type floating $P V$}

In order to rotate floating structure on water surface, a rotation center of the rotating structure is needed, and fastening at the rotation center can be effective. Therefore, in order to fix the rotation center, stakes or pillar-like object must be installed to the direction of the water. If the water depth is great, however, the structure installed to fix the rotation center reduces the economic value against the installation cost for PV power plants, and it is difficult to secure structural safety for the fixation of a horizontal-direction large-sized structure at a single point. Thus, in order to maintain rotation center and facilitate the rotation of structure, it was composed of internal rotation structure and external fixed structure. The external fixed structure performs the guide role for the internal circular structure to rotate, and is connected to the mooring system so that the entire structure can be fixed at a certain location.

The floating tracking-type PV structure shown in the Fig. 2 is expanded by connecting 4 unit structures, and the internal rectangular structure hosts the operative device for rotation and various electrical devices. Also, the external fixing structure was designed to be used as a path for maintenance being a connecting element that connects octagonal unit structures.

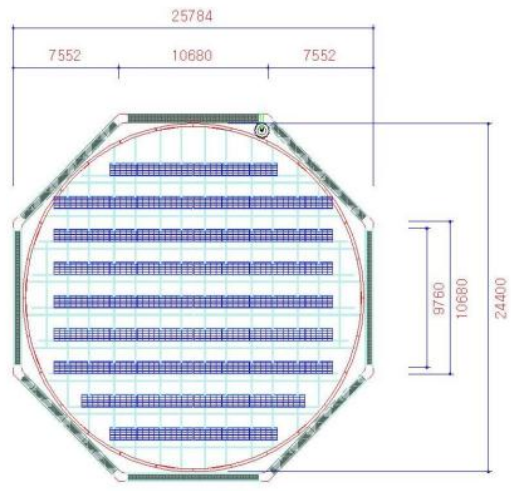

(a) 1 unit

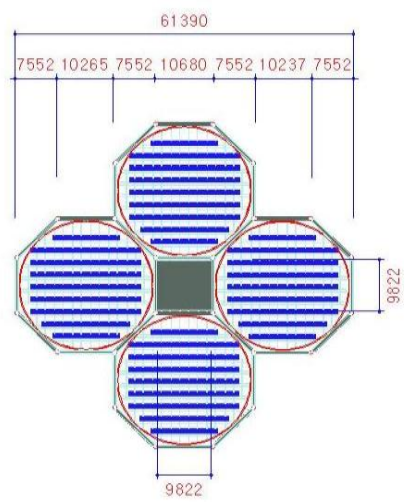

(b) expansion

Fig. 2. Tracking-type floating PV structure design and basic concept of structure expansion.

\subsection{Design concept of tracking-type floating $P V$}

1) Rotating structure for azimuth tracking

The tracking-type floating PV consists of internal rotating structure and external fixing structure, so the rotation mechanism of how the internal circular structure rotates within the external fixed structure is important. 
Such rotation mechanisms include the method using rope and forward/reverse rotation, the method using worm and worm gear, the method using chain and roller guide, the method using fixed buoyancy roller guide and chain or rope, and the method using gear and rotation ring.

Among them, the method using gear and rotation ring places the gear at the fixed buoyancy, wraps the enclosed rotation ring so that the gear is wound to the perimeter of circular buoyancy, and uses the motor; it is characteristic of attaching circular roller to the commissure of rotating buoyancy and fixed buoyancy in order to facilitate rotation while holding the rotating buoyancy to prevent movement due to water surface flow.

2) Tilt-variable structure for tracking angle of tilt

Besides tracking azimuth angle by rotation of internal circular structure, the dual-axis tracking-type floating PV which tracks angle of tilt was designed.

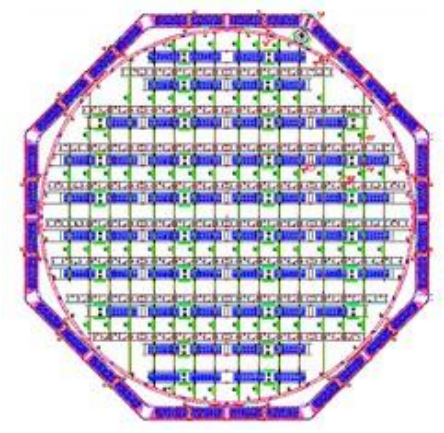

(a) Rotating structure

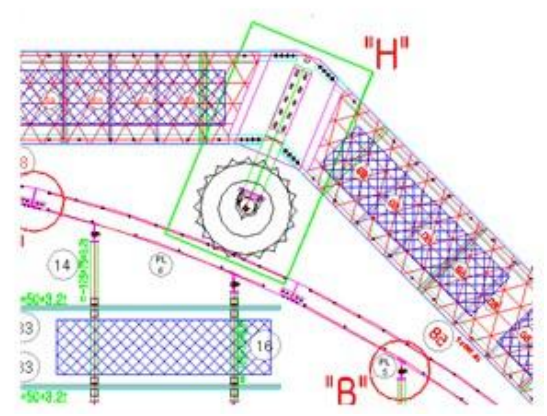

(b) Gear and rotating ring

Fig. 3. Rotating structure for azimuth tracking using gear and rotation ring.

\subsection{Tracking algorithm}

Tracking the location of sun on the ground includes the method of using optical sensor (passive), the method by astronomical calculations (active), and the method combining the two.

The sun tracking device using optical sensor involves operation of an actuator to operate the sun tracking device using the difference of radiation intensity detected through photo sensor. The sun tracking method using astronomical calculations involves using information of longitude and latitude of the tracker system installation location and performs calculation or computation by time so that it in the optimal angle and linear direction with the sun at all times.

The sun tracker using optical sensor is operated by the sensor response characteristics, and thus has the weakness of not being able to make an accurate sun detection in an environment with diffusion such as under cloudy weather, and thus tracking accurate location with astronomical calculations is used to supplement.

As the sun location information is acquired by an astronomical method, it can track the sun even when the sun does not appear for a long period of time, and the malfunction by foreign substance can be limited within a certain range, so while there is the advantage of reducing errors in sun tracking, there is the weakness that the system initial position must be accurate.

The composite-type combining these two uses the two methods intelligently based on weather information (amount of radiation, wind speed, and temperature) for increasing efficiency, and was applied to the tracking-type floating PV tracking device.

On water surface, however, unlike on ground surface, the entire structure moves and rotates by wind or other environmental variables even if the mooring device is installed, so an error may occur to the azimuth angle.

In order to compensate for this error, GPS receiver and terrestrial magnetism sensor were used to compensate for the twisted angle and the algorithm minimizing the error from external disturbance on water surface was applied. The error compensation by external disturbance is as in the following Fig. 4. 


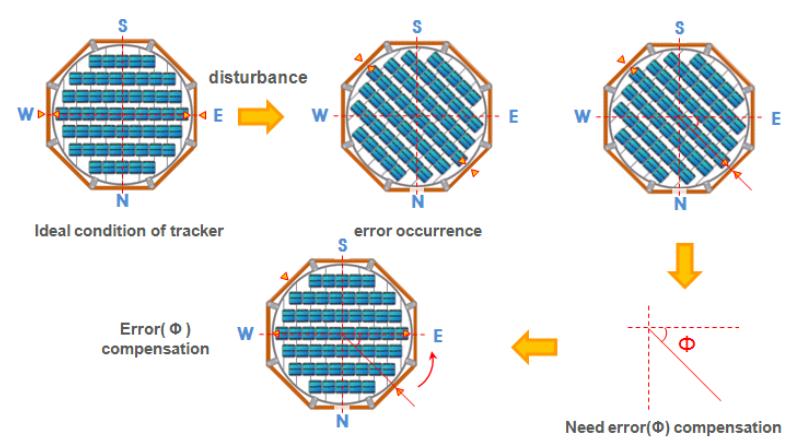

Fig. 4. Error compensation by external disturbance.

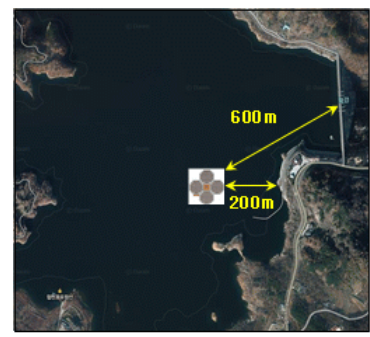

(a) Site

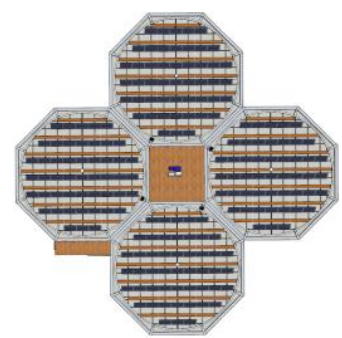

(b) Structure

Fig. 5. Prospective installation site for tracking-type floating PV demonstrative plan.

\subsection{Other system design}

Besides, $100 \mathrm{~kW}$ tracking-type floating PV to be developed in this study was designed by applying FRP-material structure, multiple sinker-type mooring system, and USN-based remote surveillance technology.

\section{Conclusion}

In this study, the optical design plan of tracking algorithm for angle of inclination and azimuth angle and the rotation mechanism to be applied for development of $100 \mathrm{~kW}$ tracking-type floating PV was described. Rotation mechanism was designed so that the rotating buoyancy does not move by the water surface flow, and the azimuth angle can be tracked in a combination of passive and active for the angle of array support.

The demonstrative plant of this study was selected by integratively considering the developing survey, site survey, solar pathfinder analysis, and influence due to gate opening during flood and current dam operation status. Currently, $100 \mathrm{~kW}$ and $500 \mathrm{~kW}$ fixed-type floating PV is to be installed at the Hapcheon dam in operation.

\section{Acknowledgements}

This research was conducted under the research fund support of the Ministry of Land, Infrastructure and Transport's Construction Technology Innovation Program (Project number: 11 technical renovation C-03, Development of ICT fusion technology for the commercialization of the floating PV system).

\section{References}

[1] Choi YK, Lee NH, Kim KJ, Cho Y. A study on the influence to solar radiation by changing the azimuth and tilt of a photovoltaic array. Journal of KIEE, 2013; 62(5).

[2] A study on research of suitable site for development and elementary technology of photovoltaic system, K-Water, 2006.

[3] A study on research of suitable site for development and elementary technology of photovoltaic system, K-Water, 2008.

[4] Groundwork research for Commercialization of Floated Photovoltaic System, K-Water, 2011. 